\title{
Reply to the letter to the editor by Mungmunpuntipantip et al.
}

\author{
M. Longo ${ }^{1,2} \cdot$ M. I. Maiorino ${ }^{1,2}\left(\mathbb{D} \cdot\right.$ K. Esposito $^{1,3}$
}

Received: 22 September 2021 / Accepted: 6 October 2021 / Published online: 22 October 2021

(C) Italian Society of Endocrinology (SIE) 2021

Dear Editor,

We are grateful to Drs. Mungmunpuntipantip and Wiwanitkit for their comments [1], and interest in our work [2]. We acknowledge that worse glucose control is an important independent risk factor for COVID-19 severity and mortality in patients with diabetes, especially in patients with disease progression and hypoxemia, who were not included in our study. We enrolled patients with type 1 diabetes and COVID-19 with asymptomatic or pauci-symptomatic course not requiring hospitalization. We found a significant worsening of the CGM-derived indices of glycemic control during the infection, which presented independently of the presence of fever. This aspect is interesting, as it suggests that potential virus-induced mechanisms may impair glucose metabolism also in pauci-symptomatic or asymptomatic patients with diabetes. Moreover, with the spread of SARS-CoV-2 around the world, the spectrum of different medical therapies to treat COVID-19 has rapidly and continually evolved and an early and adequate treatment should be performed as soon as possible. Our young patients did not warrant medical intervention, hospitalization or oxygen therapy and the disease could be managed in home setting. They were treated with antibiotics, antipyretics and vitamins supplementations. Those requiring corticosteroids therapy were excluded from the analysis, for the known potential effects of these agents on glucose levels. Alternative therapies could not be considered since we included only patients with type 1 diabetes, whose treatment is essentially based only on insulin.
However, diabetic care was ensured by telemedicine visits every 2 weeks and an insulin dose adjustment was performed at each telemedicine session. Telemedicine revealed itself as a valid and crucial tool to favor management of diabetes, during the pandemic and, hopefully, in the post-pandemic.

\section{Declarations}

Conflict of interest None to declare.

Research involving human participants and/or animals This article does not contain any studies with human participants or animals performed by any of the authors.

Informed consent For this type of study formal consent is not required.

\section{References}

1. Mungmunpuntipantip R, Wiwanitkit V (2021) Glucose control in type 1 diabetes and COVID-19. Letter to the editor. J Endocrinol Invest. https://doi.org/10.1007/s40618-021-01688-0

2. Longo M, Scappaticcio L, Petrizzo M et al (2021) Glucose control in home-isolated adults with type 1 diabetes affected by COVID19 using continuous glucose monitoring. J Endocrinol Invest. https://doi.org/10.1007/s40618-021-01669-3

Publisher's Note Springer Nature remains neutral with regard to jurisdictional claims in published maps and institutional affiliations.

M. I. Maiorino

mariaida.maiorino@unicampania.it

1 Department of Advanced Medical and Surgical Sciences, University of Campania "Luigi Vanvitelli”, Naples, Italy

2 Division of Endocrinology and Metabolic Diseases, University of Campania "Luigi Vanvitelli", Naples, Italy

3 Unit of Diabetes, University of Campania "Luigi Vanvitelli”, Naples, Italy 